\title{
Fabrication Procedure and Performance of 3D Printed X-Band Horn Antenna
}

\author{
Sebastian G. Wirth*, Ivor L. Morrow* and Ian Horsfall* \\ *Cranfield University, Laboratory of Electromagnetic Systems Engineering, \\ Shrivenham Campus, Oxfordshire, SN6 8LA, UK. \\ Email: Sebastian.Wirth@cranfield.ac.uk,I.L.Morrow@cranfield.ac.uk
}

\begin{abstract}
.
This paper presents the design, fabrication and performance of fusion deposition modeled 3D printed X-band horn antennas. The WR90 waveguide feed and pyramidal horn flare are printed as one piece from Acrylonitrile Butadiene Styrene (ABS). Different metallisation techniques are assessed to provide a uniform 40 micron coat on the ABS surfaces. Uniquely, the coaxial waveguide launcher is integrated with the waveguide section in a single interference fit operation. The measured and simulated radiation patterns showed good correlation and the antenna return loss was $\leq-10 \mathrm{~dB}$ over the 8.2$12.4 \mathrm{GHz}$ operating range. The measured and simulated antenna gain was in good agreement and increased monotonically from $10-17 \pm 1.0 \mathrm{dBi}$ across the operating frequency.
\end{abstract}

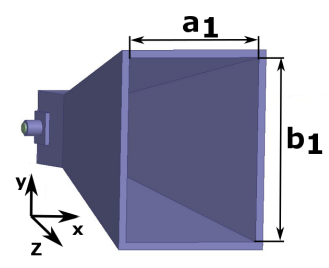

(a)

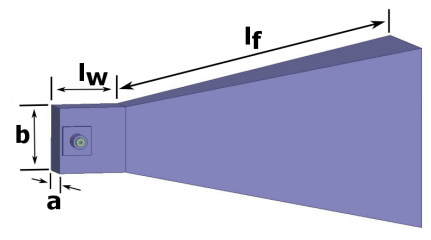

(b)

Figure 1. Geometry and finite element model for the 3D printed Pyramidal horn antenna (a) end view (b) side view.

\section{Introduction}

In recent years several ultra-wideband radar systems have been developed for many applications, such as ground penetrating radar (GPR) [1], remote sensing [2], medical diagnostics [3] and disaster relief [4]. In additional to conventional scanning techniques these systems may be operated from unmanned ground [5] or low level airborne vehicles [6] in remote regions. Light weight, low cost and ease of manufacture are attractive features for many of the antennas and microwave components used in these applications.

The cost-per-part for fusion deposition modeling (FDM) 3D printing makes it a cost effective method for prototyping and small scale custom fabricating that would otherwise require significant investment in molds or machining. Advances in the quality and resolution of additive manufacturing such as FDM and stereolithography (SLA) or resin printing is influencing many antenna engineers to consider new manufacturing and design techniques [7], [8], [9].

This paper presents the design, 3D fabrication process and RF performance of a horn antenna. A novel feature of the antenna is the antenna part is fabricated as a single ABS plastic part. This comprises the waveguide section incorporating the coaxial launcher housing and the horn antenna flare. Section 2. describes the electromagnetic design of the Pyramidal
Table 1. Dimensions of the Pyramidal antenna shown in Fig. 1 (units in mm).

\begin{tabular}{|l|l|}
\hline aperture $\left(a_{1}, b_{1}\right)$ & $(54.5,73.0)$ \\
\hline flare length $l_{f}$ & 133.0 \\
\hline waveguide $(a, b)$ & $(10.16,22.28)$ \\
\hline waveguide section length $l_{w}$ & 30.0 \\
\hline wall thickness $t$ & 3.0 \\
\hline feed diameter $d$ & 7.0 \\
\hline
\end{tabular}

horn antenna. Section 3. discuss the results of application of different metallic coatings and processes and assesses their quality. A pretuned SMA waveguide launcher is then interference fitted to the prematched waveguide section. This approach reduces prototype assembly and labour costs associated with post impedance tuning. In Section 4., the advantages of the 3D design approach are demonstrated through results for electromagnetic analysis of the horn antenna and its accuracy is verified through correlations with measurement data. Finally some conclusions are given in Section 5. and potential guidelines for repeatability and quality assessment are discussed.

\section{Antenna Design}

\subsection{Pyramidal Horn Antenna Design}

The GPR antennas were required to operate at X-Band (8.2$12.4 \mathrm{GHz}$ ) with a minimum gain of $15 \mathrm{dBi}$. Fig. 1 illustrates the geometry of the Pyramidal horn with a short waveguide section used to feed the flare. The well known procedure described in [10] was followed to determine the initial flare dimensions and aperture that met the gain criteria. An electromagnetic finite element model, shown in Fig. 1, was constructed and included many of the practical design features of the antennas such as; A sheet impedance boundary condition with no surface roughness was used to model the 40 micron thick copper coating applied to the ABS (dielectric constant $\varepsilon_{r}=2.7$ ) plastic 3D print. A $7 \mathrm{~mm}$ circular aperture was located on the broad wall of the waveguide and $8 \mathrm{~mm}$ from the back wall. 
A coaxial waveguide port of outer radius $3.5 \mathrm{~mm}$ was integrated with the circular aperture, with the inner coaxial pin $(1.27 \mathrm{~mm}$ diameter) initially projecting $8.2 \mathrm{~mm}$ into the waveguide. To complete the model of the SMA launcher a cylindrical Teflon $\left(\varepsilon_{r}=2.1, \tan \delta=0.0001\right)$ jacket was extended from the waveguide port to the boundary of the inner waveguide wall.

Numerical experiments were performed to optimise the $\left(\rho_{e}, \rho_{h}\right)$, which are defined as the lengths from the horn aperture to the imaginary apex of the horn in E- and H-planes respectively, see [10] for further details. The aim was to provide similar $3 \mathrm{~dB}$ beamwidths in both $\mathrm{E}$ - and $\mathrm{H}$-planes and reduce cross-polarised levels below $30 \mathrm{~dB}$. The optimised design dimensions are given in Table 1 and resulted in a flare length of $5.5 \lambda$ and calculated gain of $16.6 \mathrm{dBi}$ at $10 \mathrm{GHz}$.

\section{Antenna Printing and Fabrication}

\subsection{D Printing}

The horn antenna prototype was FDM printed using the Stratasys $\mu$-print SE 3D printer as one part. The print material was non-conductive white coloured Acrylonitrile Butadiene Styrene (ABS) with dielectric constant $\varepsilon_{r}=2.72$ and $\tan \delta=0.02$. ABS possess good mechanically strength, chemical inertness and can tolerate environments temperatures changes between -20 and $80^{\circ} \mathrm{C}$. At a temperature of $210^{\circ}$ the ABS filament becomes molten and is extruded from the CNC printer nozzle with a diameter of $0.4 \mathrm{~mm}$ and layer thickness $0.1 \mathrm{~mm}$ using machine standard parameters for ABS materials. The completed printed part was allowed to harden and the support structure dissolved using a Stratasys SCA-1200 (support cleaning apparatus) and WaterWorks P400SC solution that contains a weak solution of Sodium Hydroxide.

The 3D printed part was inspected by taking micrometer measurements on the accuracy and surface finish quality. While straight edge sections of the ABS waveguide and wall thickness were accurately printed $\pm 0.2 \mathrm{~mm}$; non-linear surfaces such as the horn flare, particularly in the narrow wall exhibited resolution stair-casing (parts are built layer-by-layer) effects and surface roughness. Repeatability (or tolerances) between different 3D prints showed significant variability of up to $\pm 0.5 \mathrm{~mm}$ in fine feature detail.

\subsection{Coating and Electroplating Experiments}

Conductive coatings were applied to four 3D printed Pyramidal horn antennas. The skin depth of copper at $12 \mathrm{GHz}$ is $10 \mu \mathrm{m}$ thus a coating of $40 \mu \mathrm{m}$ was considered adequate to support electromagnetic fields inside the horn antenna and contain skin depth penetration. Prior to application of conductive finishes both interior and exterior printed part surfaces were polished using P150 grade silicon carbide papers.

Solvent based spray paints containing Zinc (Nickel Screen Compound - Electrolube) and Copper (Kontakt Chemie EMV) were applied to two printed horns. Both aerosols provided coat thickness of between 30 to $50 \mu \mathrm{m}$. Coated parts were then cured in a temperature controlled environment for 12 hours. The cured coatings on the antenna had measured surface resistivity of $0.7 \Omega / \mathrm{sq}$ for the Zinc finish and $20 \Omega / \mathrm{sq}$ for the copper finish. A third 3D printed horn had $50 \mu \mathrm{m}$ thick copper tape cut to shape and fixed to the inner surfaces of the horn and waveguide. The seams of the copper tape were electrically bonded together using a silver paint that was allowed to set and the surface hand polished. Finally, a fourth test piece was commercially electroplated with a $40 \mu \mathrm{m}$ copper coating. Fig. 2 shows the four horn antenna apertures with the different conductive finishes applied.
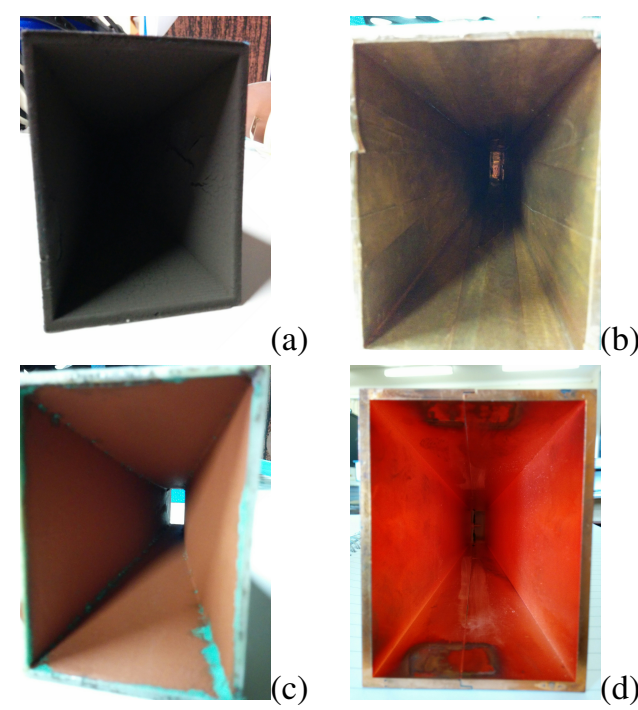

Figure 2. Horn antenna apertures showing different conductive surface finishes applied (a) Zinc, (b) Copper tape, (c) Copper spray and (d) Copper electroplating.

While Zinc and Copper spray was relatively easy to apply in spray form it did not spread evenly over the ABS part. In particular, the Zinc spray coating brittled as it dried and eventually cured with significant surface resistance. In contrast the Copper spray adhered well and provided a fairly even coat over flat surfaces. The Copper tape adhered well to the ABS parts but access was difficult to polish internal electrically bonded seems and corners. Copper electroplating the 3D printed part provided an even coverage and required little surface polishing. Three new horn antennas were 3D printed and electroplated and used in the subsequent work.

\section{Results and Discussion}

\subsection{Impedance Match and Coating Performance}

An SMA coaxial probe with interference fit tolerance was inserted into the waveguide aperture to excite the $T E_{01}$ mode. The probe was located $\lambda / 4$ from the back wall of the waveguide and the probe length determined from simulation to provide the optimum impedance match. Fig. 3 shows the simulated and measured reflection coefficient for the probe length $L p=9.6 \pm 0.2 \mathrm{~mm}$. A return loss of $\leq-10 \mathrm{~dB}$ was achieved experimentally across the X-band. The resonance matching behaviour predicted from simulation was damped and this 


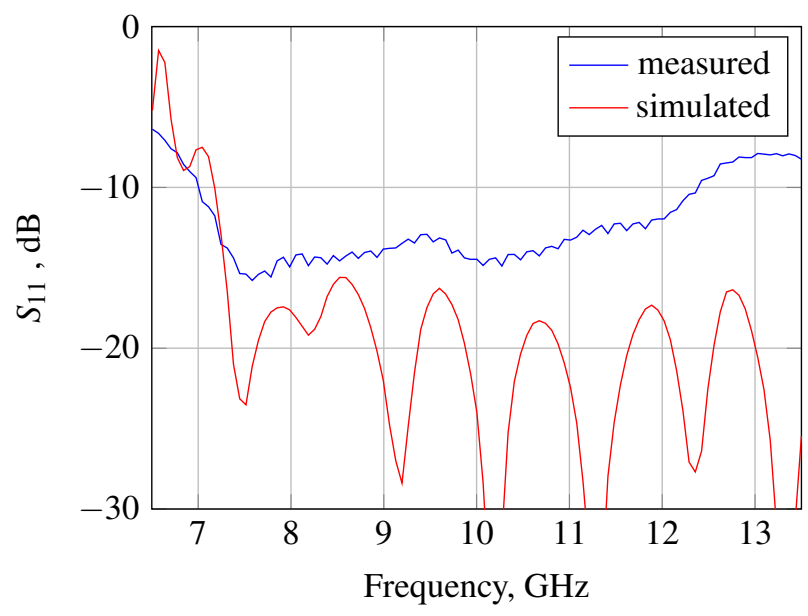

Figure 3. Simulated and measured reflection coefficient response $S_{11}$ for optimum probe length $L_{p}$.

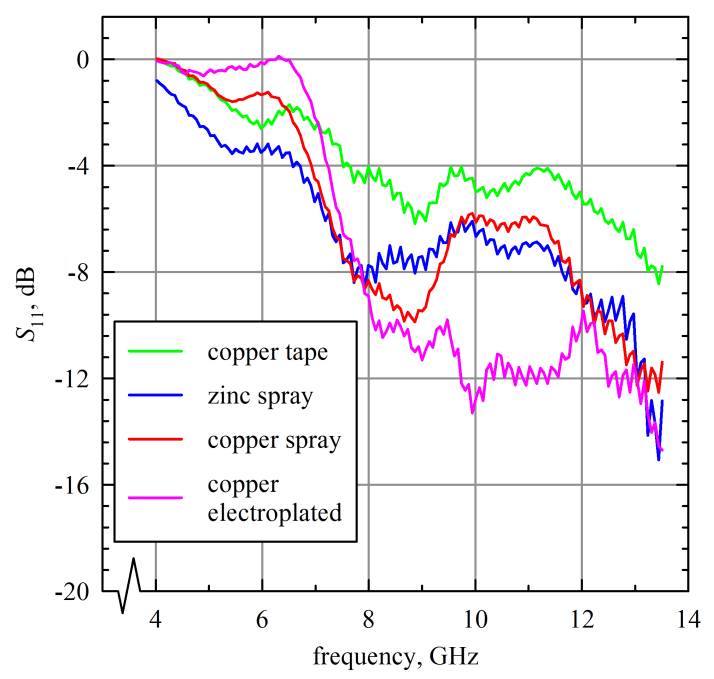

Figure 4. Measured reflection coefficient responses $S_{11}$ for the Pyramidal horn for different metal coating applied.

was traced to 3D print tolerance issues in the circular aperture dimension of the coaxial housing were the SMA coaxial launcher was integrated.

The performance of the different coatings on the $3 \mathrm{D}$ printed antennas was qualitatively assessed by measuring the $S_{11}$ reflection coefficient for the horn antennas. It was thought this would give a measure of the coating uniformity and surface resistance of the coatings. The optimum matched probe length was used for all antennas and Fig. 4 shows the results. It is evident that the copper electroplated horn provided the best match and most uniform coating.

\subsection{Gain and Radiation Field Patterns}

The boresight measured gain is calculated using Eqn. 1 [10] which assumes the antennas are polarisation matched and the symbols have there usual meaning. Frequency dependent cable losses $L_{\text {cable }}$ and individual transmit $\left(1-\left|\Gamma_{t}\right|^{2}\right)$ and receive $\left(1-\left|\Gamma_{r}\right|^{2}\right)$ impedance mismatch losses are taken account off.

$$
P_{r}=P_{t}\left(\frac{\lambda}{4 \pi R}\right)^{2} G^{2} L_{\text {cable }}\left(1-\left|\Gamma_{t}\right|^{2}\right)\left(1-\left|\Gamma_{r}\right|^{2}\right) \quad\left|\hat{\mathbf{a}}_{\mathbf{t}} \cdot \hat{\mathbf{a}}_{\mathbf{r}}\right|
$$

The measured and simulated gain versus frequency of the horn antenna is shown in Fig. 5. The plot shows a gain response of over $15.1 \mathrm{dBi}$ for frequencies above $8.2 \mathrm{GHz}$ with a peak gain of $17 \mathrm{dBi}$ at $12.4 \mathrm{GHz}$.

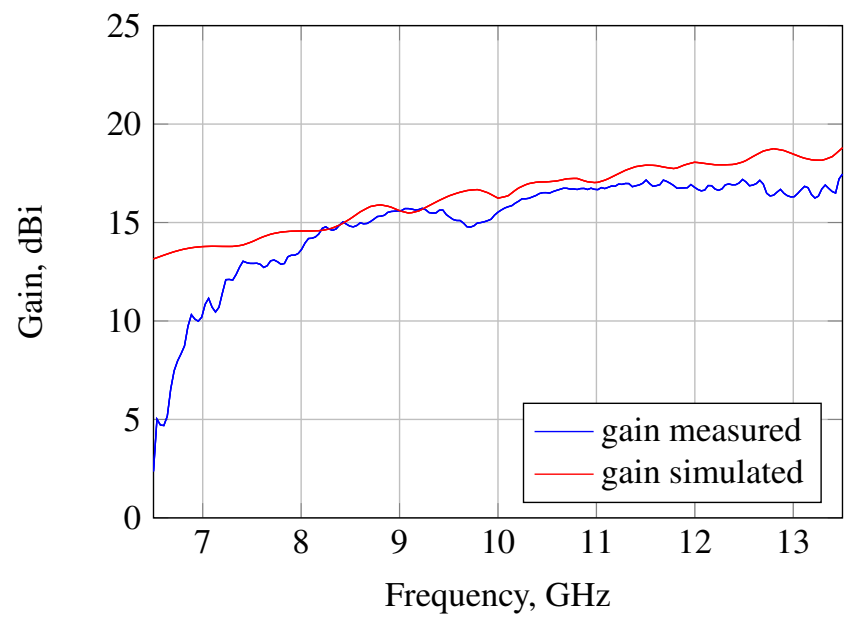

Figure 5. Measured and simulated gain characteristics of the $3 \mathrm{D}$ printed Pyramidal horn antenna.

The measured and simulated E-plane and H-plane radiation patterns for the fabricated prototype antenna are presented in Fig. 6 for different frequencies of operation. The radiation patterns show good correlation between the measured and simulated co-polarised fields in both planes. The measured crosspolar field levels are below $-25 \mathrm{~dB}$, while simulated levels were below $30 \mathrm{~dB}$. The average half-power beamwidth for the Eplane and H-plane co-polar patterns were $28^{\circ}$ and $30^{\circ}$, respectively with a peak measured gain of $15 \mathrm{dBi}$ and $16.6 \mathrm{dBi}$ calculated at $10 \mathrm{GHz}$. The radiation efficiency was calculated from simulation to be $\geq 96 \%$.

\section{Conclusions}

This paper describes the 3D print fabrication process for a bespoke Pyramidal X-band horn antenna for GPR applications. The antenna is fabricated as one part and a pre-matched SMA coaxial probe is push fitted to the waveguide launcher. Copper electroplating provided the most uniform metal coating. Simulations predicted a return loss of $\leq-15 \mathrm{~dB}$ impedance match; however due to FDM 3D printing tolerances (at the coaxial launcher aperture) a return loss of only $\leq-10 \mathrm{~dB}$ was achieved. A better practice could be to solid print the coaxial housing waveguide wall and post 3D printing precision drill the coaxial aperture. Similarly the measured antenna gain of $\geq 15 \mathrm{dBi}$ was $1 \mathrm{~dB}$ down on the calculated value. The measured and simulated antenna field patterns showed good correlation and the measured cross-polar power levels were all $\leq-25 \mathrm{~dB}$. The resultant antenna is lightweight $(60 \%$ lighter 


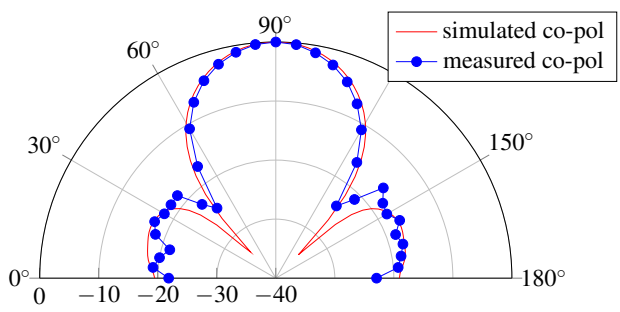

(a) E-plane, $8 \mathrm{GHz}$

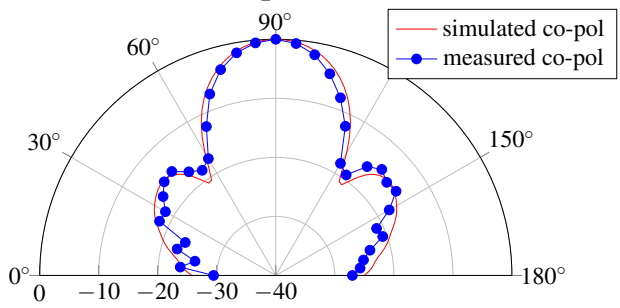

(c) E-plane, $10 \mathrm{GHz}$

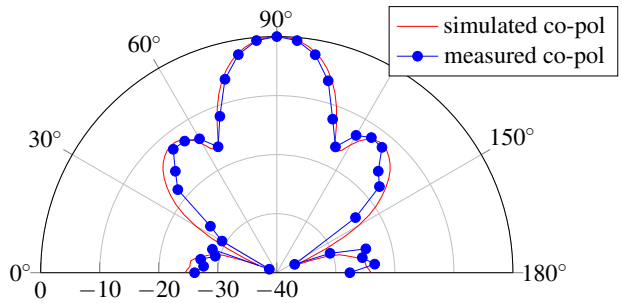

(e) E-plane, $12 \mathrm{GHz}$

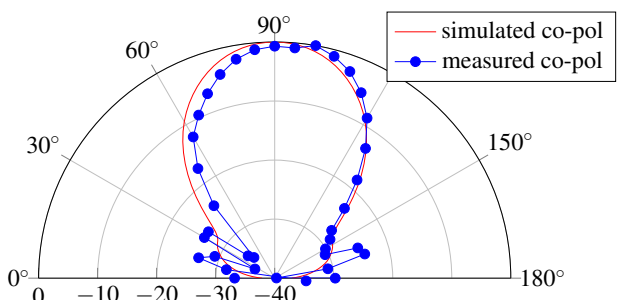

(b) H-plane, $8 \mathrm{GHz}$

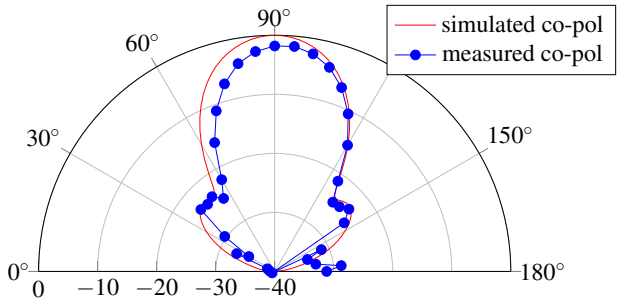

(d) H-plane, $10 \mathrm{GHz}$

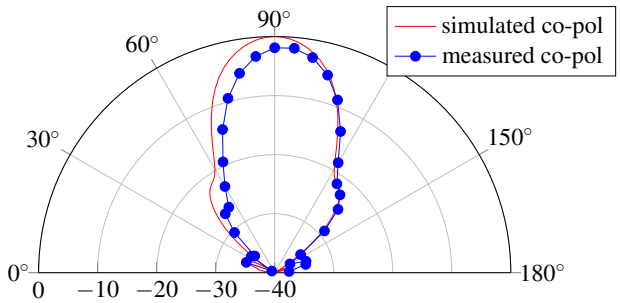

(f) H-plane, $12 \mathrm{GHz}$

Figure 6. Measured and simulated principal E-plane and H-plane radiation patterns for the 3D printed horn antenna (E-plane (xoz) and H-plane (yoz), as depicted in Fig. 1), and •- • indicates measured co-polar field pattern and - indicates simulated co-polar field pattern.

than an equivalent metal cast antenna), low cost, and feasible to manufacture at remote locations. However, the antenna is vulnerable to light impact and mechanical damage and should be handled with care.

\section{Acknowledgment}

The authors thank the Find A Better Way charity for their support of this research under the DETERMINE programme (grant number 2015/001D).

\section{References}

[1] J. Jeov and S. Lambot and A. Fedeli and A. Randazzo, Groundpenetrating radar for tree trunk investigation,9th International Workshop on Advanced Ground Penetrating Radar (IWAGPR), pp. 1-6, (2017).

[2] S. C. Steele-Dunne and H. McNairn and A. Monsivais-Huertero and J. Judge and P. W. Liu and K. Papathanassiou, Radar Remote Sensing of Agricultural Canopies: A Review, IEEE Journal of Selected Topics in Applied Earth Observations and Remote Sensing, vol. 10, no. 5, pp. 2249-2273, (2017).

[3] S. Sarjoghian and Y. Alfadhl and X. Chen, Compact ultrawideband double-ridged horn antennas for medical imaging, Loughborough Antennas Propagation Conference (LAPC), pp. 1-4, (2016).

[4] Q. An and Z. Li and F. Liang and M. Liu and J. Wang, Study on the detection performance of UWB bio-radar with segmented time window, CIE International Conference on Radar (RADAR), pp. 1-5, (2016).
[5] W. S. Benedix and D. Plettemeier and K. Wolf and V. Ciarletti and S. E. Hamran and C. Corbel, External calibration of GPR antenna accommodated on a rover Proceedings of the Fourth European Conference on Antennas and Propagation, pp. 1-4, (2014).

[6] K. V. Hoel and S. Kristoffersen and J. Moen and G. Holm and T. S. Lande, Characterization of a 3D printed wideband waveguide and horn antenna structure embedded in a UAV wing, 10th European Conference on Antennas and Propagation (EuCAP), pp. 1-4, (2016).

[7] K. V. Hoel and S. Kristoffersen and J. Moen and K. G. Kjelgrd and T. S. Lande, Broadband antenna design using different 3D printing technologies and metallization processes, 10th European Conference on Antennas and Propagation (EuCAP), pp. 1$5,(2016)$

[8] E. G. Geterud and P. Bergmark and J. Yang, Lightweight waveguide and antenna components using plating on plastics, 7th European Conference on Antennas and Propagation (EuCAP), pp. 1812-1815, (2013).

[9] K. V. Hoel and T. Hellum and S. Kristoffersen, High power properties of 3D printed antennas, IEEE International Symposium on Antennas and Propagation (APS-URSI), pp. 823-824, (2016).

[10] C.A. Balanis, Antenna Theory, Wiley, ISBN:0-471-66782-X, Chap. 13, (2005). 